\title{
I-V Characteristics of Cadmium Telluride Quantum Dots Diode Fabricated by Drop Casting Method
}

\author{
Meera R Gumaste \\ Department of Physics \\ Dayananda Sagar College of Engineering \\ Bangalore, India \\ VTU-RRC \\ Belagavi, India \\ dixit.meera11@gmail.com
}

\author{
Gururaj A Kulkarni \\ Department of Physics \\ UBDT College of Engineering \\ Davanagere, India \\ gakulkarni1@gmail.com
}

\begin{abstract}
Current (I)-Voltage (V) characterizations of a CdTe quantum dot diode is discussed in this article. The quantum dot diode is fabricated by a simple drop casting method on an n-type Indium Tin Oxide glass substrate. The linear variation of current with respect to applied voltage can be attributed to reduced grain boundary defects and enriched crystallinity. It is also observed that quantum dots can show good current conduction than CdTe nano-rods.
\end{abstract}

Keywords: CdTe Quantum dot diode, ITO, I-V characteristics, grain boundary defects

\section{INTRODUCTION}

Semiconductor nanocrystals known as Quantum Dots (QDs) have attracted remarkable consideration in the fabrication of devices such as optoelectronic, photovoltaic and photoluminescent devices [1] due to their size dependent physical and chemical properties [2]. II-VI semiconductors possess such properties which are suitable for device fabrications [3]. Cadmium Telluride (CdTe) is one such IIVI compound semiconductor which is widely used in the fabrication of solar cell $[4,5]$, Schottky thin film solar cell [6] made from CdTe nanocrystals. However making any device requires a sophisticated technique for its better performance. So far in the literature heterojunction diodes [7], CdTe based QD sensitized solar cells [5], CdTe nanowire thin film solar cells [8], CdTe QD electrodes [9] are extensively studied. However work emphasized on CdTe QD diodes are sparse.

In the present work authors have made an attempt to assemble a simple laboratory made $\mathrm{p}-\mathrm{n}$ junction diode using CdTe QDs. In that direction initially subsequent sections puts light on the structure and conductivity of the as synthesized CdTe QDs as a contextual work. Later the simple fabrication process is explained with the device layout. I-V characteristics of the QDs diode is discussed. Also further improvements needed in the device fabrication and other possibilities of improvement in the performance are discussed at the end of this article.

\section{BACKGROUND WORK ON CDTE QDS:}

As the work presented here focusses mainly on diode fabrication and its I-V characteristics, other characterizations such as Powder- X-ray Diffractometer and van der Pauw Hall measurement for the study of electrical parameters including synthesis are discussed by the authors somewhere else [10]. However for the completeness of the information about the material, method of synthesis and properties like structure, size and conductivity are mentioned in the present article. To begin with CdTe QDs are synthesized by single injection hydrothermal (SIH) method described by Jai et.al [11]. SIH synthesis technique is a less expensive technique which can be done in a normal chemistry laboratory. It comprises of injecting Te precursor in the form of $\mathrm{NaTeO}_{3}$ in $\mathrm{Cd}$ precursor. Later the samples are collected systematically for different duration of time like 15, 30 and 45 minutes. However for the fabrication of a QD diode, CdTe QDs synthesized for 15 minutes have been used. The chosen CdTe QDs are of hexagonal crystal structure with particle size $3.66 \mathrm{~nm}$. The electrical parameters are also studied which manifests that these QDs are of p-type conductivity.

\section{FABRICATION OF CDTE QD DIODE:}

The device layout of the QD diode is shown in fig. 1. The active layer in the diagram refers to CdTe QDs. For this, the as synthesized CdTe QDs are sonicated for 30 minutes in sonication bath. Then the QDs are re-dispersed in pyridine after filtering all the deionized water content from the solution containing QDs. Again QDs are sonicated in pyridine to get the uniform distribution of QDs. An n-type Indium Tin oxide (ITO) conductive glass slides of $2.5 \mathrm{~mm} \mathrm{X}$ $2.5 \mathrm{~mm} \times 1.1 \mathrm{~mm}$ dimensions with resistivity $\leq 20 \mathrm{ohms} / \mathrm{sq}$ is used as a substrate. Further a simple drop casting method is employed to deposit the QDs on ITO substrate. For this $300 \mu$ l of QDs solution is taken and drop casted on the ITO substrate using a micro-pipette. The ITO substrate is then annealed at $100^{\circ} \mathrm{C}$ for one hour. After annealing, the side of ITO coated with QDs turns black indicating successful deposition of CdTe QDs on the ITO substrate and QD film is formed. At this point the assembly is called as a diode made from CdTe QDs.

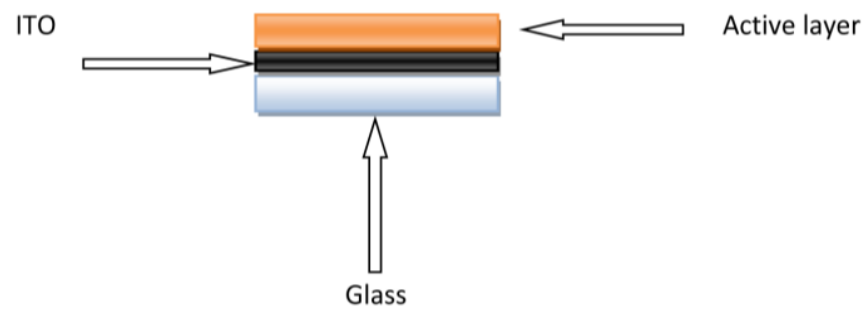

Fig. 1. QD diode layout

\section{I-V CHARACTERISTICS OF QD DIODE:}

For the current-voltage characteristics of a QD diode, DC Probe station PM 5 attached with Agilent Device Analyzer B 1500 A with pulsed source $5 \mathrm{MHz}$ and $0.1 \mathrm{fA} / 0.5$ 
microvolts measurement resolution is used. The DC voltage between $0 \mathrm{~V}$ to $1 \mathrm{~V}$ in the step of $0.01 \mathrm{~V}$ is applied to the diode. A linear and symmetric variation in current is observed with respect to voltage varied between $0 \mathrm{~V}$ to $1 \mathrm{~V}$ as it is evident from fig. 2. The linear and symmetric behavior of the I-V characteristics indicates uniform size of the QDs. The interconnection between the particles is well established [12] and distribution of QDs is uniform throughout the QD film. The current across the diode is less probably due to nano-cracks [13] and grain boundary defects [14] formed during the deposition. However the current observed across the QDs is higher compared to CdTe nanorods [15]. Consequently it can be said that QDs can show good current conduction than the rod shaped morphology. This indicates that the grain boundary defects which impedes the current conduction is minimized in CdTe QDs than in CdTe nano-rods and enriched crystallinity in QDs can be seen. The decrease in the grain boundary defect may be due to reduced particle size in QDs.

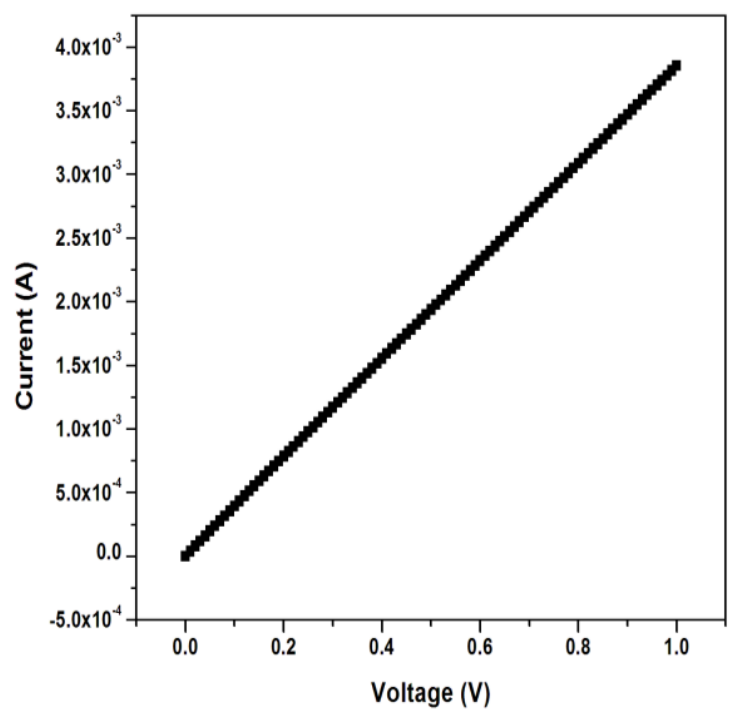

Fig. 2. I-V characteristics of CdTe QD diode

Further to it can be said that such a QD diode when devised with a Schottky barrier a QD film solar cell can be formed [6].

\section{CONCLUSION}

Hexagonal structured CdTe QDs are synthesized by single injection hydrothermal method. The QD diode is fabricated by drop casting method of CdTe QDs on ITO glass substrate. Linear I-V characteristics of the QD diode signifies the good interconnection established between the QDs and reduce grain boundary defects. The QD diode is found to conduct good current than CdTe Nano-rods.

\section{ACKNOWLEDGEMENT}

Meera Ramachandra Gumaste is thankful to INUP, CeNSE, IISc, Bangalore, India for providing an opportunity to conduct our research work including characterizations through INUP participation. I am also thankful to Ms. Suma (CeNSE) and INUP, CeNSE where major characterizations were performed, funded by Department of Electronics and Information Technology (DeitY), Govt. of India and located at Indian Institute of Science, Bangalore, India. MRG is also thankful to Management, Principal and HOD, Department of Physics of Dayanandasagar College of Engineering, Bangalore for their constant support to carry out this research.

\section{REFERENCES}

[1] Ling Chen et.al, Sci. Adv. Mater. 2012, Vol. 4, No. 2, doi:10.1166/sam.2012.1288

[2] Hokyeong Shin et.al, Bull. Korean Chem. Soc. 2014, Vol. 35, No. 10 2895 http://dx.doi.org/10.5012/bkcs.2014.35.10.2895

[3] Sandeep Kumar et.al, CHEM. COMMUN. , 2003, 2478-2479

[4] Anjali Thakur et.al, IOSR Journal of Engineering, ISSN (e): 22503021, ISSN (p): 2278-8719 Vol. 08, Issue 6 (June. 2018), |V (VII) \| PP 16-22

[5] Guo-Yu Lan et.al, J. Mater. Chem., 2009, 19, 2349-2355 | 2349

[6] Shi Sun et.al, J. Mater. Chem., 2012, 22, 19207

[7] Nur Efsan Koksal et.al, Physica B 600 (2021) 412599, https://doi.org/10.1016/j.physb.2020.412599

[8] Sai Guduru et.al, 37th IEEE Photovoltaic Specialists Conference, Seattle, WA, 2011, pp. 002709-002714.

[9] Zusing Yang et.al, Solar Energy Materials \& Solar Cells 94 (2010) 2046-2051, doi:10.1016/j.solmat.2010.06.013

[10] Meera Ramachandra Gumaste et.al, https://doi.org/10.1016/j.matpr.2020.08.005

[11] B. Jai Kumar etal, Journal ofLuminescence178(2016)362-367, http://dx.doi.org/10.1016/j.jlumin.2016.06.012

[12] Jayakrishna Khatei and K.S.R Koteswara Rao, AIP Advances 1, 042166 (2011) http://doi.org/10.1063/1.3669408

[13] Subhash Chander et.al, Physica E 89 (2017) 29-32, http://dx.doi.org/10.1016/j.physe.2017.02.002

[14] Subhash Chander et.al, Thin Solid Films 625 (2017) 131-137, http://dx.doi.org/10.1016/j.tsf.2017.01.052

[15] Meera Ramachandra Gumaste et al 2019 IOP Conf. Ser.: Mater. Sci. Eng. 577012169 